\title{
NGC 6778: a disrupted planetary nebula around a binary central star ${ }^{\star}$
}

\author{
M. A. Guerrero ${ }^{1, \star \star}$ and L. F. Miranda ${ }^{2,3}$ \\ 1 Instituto de Astrofísica de Andalucía (IAA-CSIC), Glorieta de la Astronomía, s/n, 18008 Granada, Spain \\ 2 Consejo Superior de Investigaciones Científicas, c/ Serrano 117, 28006 Madrid, Spain \\ e-mail: mar@iaa.es,1fm@iaa.es \\ 3 Departamento de Física Aplicada, Facultade de Ciencias, Campus Lagos-Marcosende s/n, Universidade de Vigo, 36310 Vigo, Spain
}

Received 20 August 2011 / Accepted 12 January 2012

\begin{abstract}
The planetary nebula (PN) NGC6778 harbors a binary central star with a short orbital period and displays two systems of fast collimated outflows. To assess the influence of the evolution through a common-envelope phase of the binary system of NGC 6778 on its formation and shaping, we have used narrow-band images and high-dispersion long-slit spectra of the nebula to investigate its detailed morphology and kinematics. We find that the overall structure of NGC 6778 can be described as a bipolar PN. The equatorial ring is highly disrupted and many radial features (filamentary wisps and cometary knots) also show strong dynamical effects. There are clear connections between the bipolar lobes and the fast collimated outflows: the collimated outflows seem to arise from bright knots at the tips of the bipolar lobes, whereas the kinematics of the bipolar lobes is distorted. We suggest that the interaction of the fast collimated outflows of NGC 6778 with its nebular envelope has resulted in the disruption of the nebular shell and equatorial ring.
\end{abstract}

Key words. planetary nebulae: general - planetary nebulae: individual: NGC 6778 - ISM: jets and outflows binaries: close

\section{Introduction}

Planetary nebulae $(\mathrm{PNe})$ consist of stellar material ejected by stars with initial masses $\leq 8-10 M_{\odot}$. When such a star evolves off the asymptotic giant branch (AGB), it experiences copious mass loss episodes that dramatically reduce the stellar envelope. When the stellar core is finally exposed, the stellar effective temperature rises and, by the time it exceeds $30000 \mathrm{~K}$, the strong stellar UV radiation ionizes the circumstellar material and a new $\mathrm{PN}$ is born.

Our canonical view of the formation of PNe is based on the interacting stellar winds model in which the nebular shape is mostly determined by the interaction of the current fast, tenuous stellar wind of its central star (e.g., Patriarchi \& Perinotto 1991) with the previous slow, dense wind of the AGB phase (Kwok 1983). This model accounts for the gross morphology of PNe: a spherical symmetric AGB wind will result in a spherical PN, while an azimuthal density gradient in the AGB wind

* Based on observations made with the Nordic Optical Telescope (NOT) and the Italian Telescopio Nazionale Galileo (TNG) on the island of La Palma in the Spanish Observatorio del Roque de los Muchachos of the Instituto de Astrofísica de Canarias (IAC). NOT is operated jointly by Denmark, Finland, Iceland, Norway, and Sweden. TNG is operated by the Fundación Galileo Galilei of the INAF (Istituto Nazionale di Astrofisica). The data presented here were obtained in part with ALFOSC, which is provided by the Instituto de Astrofísica de Andalucía (IAA) under a joint agreement with the University of Copenhagen and NOTSA.

$\star \star$ Visiting astronomer, Cerro Tololo Inter-American Observatory, National Optical Astronomy Observatory, which are operated by the Association of Universities for Research in Astronomy, under contract with the National Science Foundation. will result in an elliptical or bipolar PN, depending on the degree of the density gradient (Balick 1987). The observations of PNe have shown that their morphologies and detailed structures are extremely rich, including small-scale nebular features (e.g., NGC 7662; Perinotto et al. 2004), collimated bipolar outflows (e.g., He 2-90; Sahai \& Nyman 2000; Guerrero et al. 2001), and point-symmetric bubbles (e.g., Hen 2-47 and M 1-37; Sahai 2000) and collimated outflows (e.g., NGC 6884 and Hen 3-1475; Borkowski et al. 1997; Miranda et al. 1999a; Riera et al. 2003). This large body of observations reinforces the idea that the interacting stellar winds models need to incorporate additional physical processes (Balick \& Frank 2002).

Among the new physical processes to take into account, fast collimated outflows (jets) or collimated fast stellar winds can be expected to have profound effects on the shaping of PNe (Sahai \& Trauger 1998). High-velocity bipolar jets impinging on the nebular material will transfer momentum and deposit kinetic energy into the nebula and break the spherical symmetry of the AGB wind. The prolonged action of collimated outflows may bore through the AGB wind and form extended cavities (e.g., $\mathrm{OH} 231.8+4.2$; Bujarrabal et al. 2002) whose orientation may change with the precession or rotation of the collimated outflow (e.g., NGC 6881; Guerrero \& Manchado 1998; Kwok \& Su 2005). The strong shock of a fast collimated outflow may have dramatic effects in the nebula itself, causing the complete disruption of the nebular shell.

The mechanisms that generate fast collimated outflows in PNe are still an unsolved question (Cantó et al. 1988; Frank et al. 1996; García-Segura et al. 2005; Nordhaus et al. 2007; Blackman 2009). Accretion disks formed during the commonenvelope $(\mathrm{CE})$ phase in a binary system have been proposed to 
be able to collimate fast outflows in PNe (Morris 1987; Livio 2000; Soker 2004; Nordhaus \& Blackman 2006), but compelling evidence of the association between close-binary nuclei and extremely asymmetric morphologies and collimated outflows has been lacking (Bond \& Livio 1990). Deeper observations are nowadays strengthening this relationship (Miszalski 2012), and certainly the study of the detailed morphology and kinematics of $\mathrm{PNe}$ with binary central stars is of great interest.

Very recently, Miszalski et al. (2011) have revealed the binary nature of the central star of NGC 6778 (PN G034.5-06.7). The short orbital period, $\sim 0.15$ days, made them conclude that it has undergone a CE phase. They also suggest the correlation between the binary evolution and the development of chaotic morphologies and fragmented shells and rings with wispy structures reminiscent of nova explosions.

The morphology and kinematics of this PN had received little attention in the past. The first narrow-band $\mathrm{H} \alpha+[\mathrm{N}$ II $]$ and [O III] images of NGC 6778 presented by Schwarz et al. (1992) hinted at an S-shaped feature protruding from an irregular shell. The deeper narrow-band $\mathrm{H} \alpha,[\mathrm{N}$ II] , and [O III] images presented by Maestro et al. (2004, hereafter Paper I) confirmed and revealed greater details of this S-shaped feature, but also disclosed an additional linear jet-like feature protruding from the central nebula along a different position angle. The morphology of these features is highly indicative of fast outflows, a suggestion that was confirmed by the radial velocities of $\sim 100 \mathrm{~km} \mathrm{~s}^{-1}$ revealed by long-slit echelle spectroscopic observations (Paper I). Owing to projection effects, the expansion velocity of these collimated outflows is certainly greater than $100 \mathrm{~km} \mathrm{~s}^{-1}$. Notably, the radial velocity in the collimated outflows increases with the radius, as would be expected for the free expansion following a sudden episode of ejection. Higher resolution narrow-band images of NGC 6778 described in the framework of a preliminary study of bipolar PNe at sub-arcsec scales (Miranda et al. 2010, hereafter Paper II) revealed a bipolar structure of the nebular shell and a complex relationship between this shell and the fast collimated outflows.

In this paper, we revisit the kinematical data introduced in Paper I in conjunction with the narrow-band images of higher spatial resolution of Paper II to investigate the spatio-kinematical structure of the nebula, and to identify the radial velocity of individual morphological features. We describe the observations in Sect. 2 and analyze the nebular morphology and kinematics and the central star near-IR properties in Sect. 3. The results are discussed in Sect. 4 and the conclusions are presented in Sect. 5.

\section{Observations}

\subsection{Narrow-band optical imaging}

Narrow-band images of NGC 6778 have been acquired on 2005 August and 2008 August using ALFOSC (Andalucia Faint Object Spectrograph and Camera) at the $2.56 \mathrm{~m}$ Nordic Optical Telescope (NOT) of the Observatorio de Roque de los Muchachos (ORM, La Palma, Spain). The detector was an EEV $2048 \times 2048$ CCD with a pixel size of $13.5 \mu \mathrm{m}$, plate scale of 0.19 pixel $^{-1}$, and field of view $(\mathrm{FoV}) 6.5 \times 6.5$. The images were obtained through narrow-band filters that isolate the $\mathrm{H} \alpha$ $\left(\lambda_{\mathrm{c}}=6568 \AA, F W H M=8 \AA\right),[\mathrm{N}$ II $] \lambda 6583\left(\lambda_{\mathrm{c}}=6589 \AA\right.$, $F W H M=9 \AA)$, and [O III] $\lambda 5007\left(\lambda_{\mathrm{c}}=5007 \AA, F W H M=8 \AA\right)$ emission lines. Table 1 lists the number of individual frames acquired for each filter, their exposure time, and the total integration time. The data were bias-subtracted and flat-fielded by
Table 1. NOT narrow-band observations.

\begin{tabular}{llccc}
\hline \hline Date & Filter & $\begin{array}{c}\text { Number of } \\
\text { frames }\end{array}$ & $\begin{array}{c}\text { Frame } \\
\text { exp. time } \\
\text { (s) }\end{array}$ & $\begin{array}{c}\text { Total } \\
\text { exp. time } \\
\text { (s) }\end{array}$ \\
\hline August 2005 & $\mathrm{H} \alpha$ & 2 & 300 & 600 \\
August 2005 & {$\left[\mathrm{N}_{\mathrm{II}}\right]$} & 2 & 300 & 600 \\
August 2005 & {$\left[\mathrm{O}_{\mathrm{III}}\right]$} & 1 & 300 & 300 \\
August 2008 & $\mathrm{H} \alpha$ & 2 & 450 & 900 \\
August 2008 & {$[\mathrm{N}$ II $]$} & 2 & 450 & 900 \\
\hline
\end{tabular}

twilight flats using standard IRAF $^{1}$ routines. We reproduce in Fig. 1 the [O III] image of NGC 6778 from the 2005 run, and the $\mathrm{H} \alpha$ and $[\mathrm{N}$ II] images from the 2008 run. The spatial resolution, as determined from the FWHM of stars in the FoV, was 0.9 for the 2005 run and 0.75 for the 2008 run. A preliminary description of these images was presented in Paper II.

\subsection{Near-IR imaging}

Near-IR images of NGC 6778 were obtained on 2003 September 19 with the Telescopio Nazionale Galileo (TNG) also at the ORM observatory. We used the NICS camera in the small field mode with a Rockwell $\mathrm{HgCdTe} 1024 \times 1024$ array. The spatial scale on the detector is 0 . $^{\prime} 13 \mathrm{pixel}^{-1}$. Images were obtained through standard $J, H$, and $K^{\prime}$ filters with total exposure times of $1440 \mathrm{~s}$ in $J$ and $H$, and $360 \mathrm{~s}$ in $K^{\prime}$. The spatial resolution, determined by the FWHM of field stars, is 0 . $^{\prime} 70-0$ ? $^{\prime} 75$. The images were reduced following standard procedures within the MIDAS package and are shown in Fig. 2.

\subsection{Optical long-slit high-dispersion spectroscopy}

High-dispersion optical spectra of NGC 6778 were obtained on 2002 June 23-24 using the echelle spectrograph on the $4 \mathrm{~m}$ Blanco telescope of the Cerro Tololo Inter-American Observatory (CTIO). The spectrograph was used with the 79 line $\mathrm{mm}^{-1}$ echelle grating and the long-focus red camera, yielding a reciprocal dispersion of $3.4 \AA \mathrm{mm}^{-1}$. The SITE $2 \mathrm{~K}$ \#6 CCD was used as detector. Its pixel size of $24 \mu \mathrm{m}$ corresponds to a spatial scale of 0.26 pixel $^{-1}$ and a spectral scale of $0.08 \AA$ pixel $^{-1}$. The echelle spectrograph was used in singleorder, long-slit mode by using a narrow-band filter that isolates the order covering the $\mathrm{H} \alpha$ and $[\mathrm{N}$ II] $\lambda \lambda 6548,6583$ lines. The slit length was $\sim 3^{\prime}$, while its width varied between $00^{\prime} 8$ and $11^{\prime \prime} 2$ for a spectral resolution $\sim 8 \mathrm{~km} \mathrm{~s}^{-1}$.

To register different nebular features of interest, the slit was oriented at position angles (PA) $15^{\circ}, 24^{\circ}, 47^{\circ}$, and $100^{\circ}$ (measured following the standard convention east of north), as illustrated in Fig. 3. The integration time of the spectra was $1200 \mathrm{~s}$ each. The spectra were wavelength-calibrated with a Th-Ar arc lamp to an accuracy of $\pm 0.4 \mathrm{~km} \mathrm{~s}^{-1}$. The radial velocity of the source was measured relative to the systemic velocity, from which we deduce a value $V_{\mathrm{LSR}}=106 \pm 2 \mathrm{~km} \mathrm{~s}^{-1}$ based on the average of the blue and red components of the radial velocity at the central position. This value implies a heliocentric velocity of $90 \pm 2 \mathrm{~km} \mathrm{~s}^{-1}$, in excellent agreement with the value of $91 \pm 3 \mathrm{~km} \mathrm{~s}^{-1}$ reported by Durand et al. (1998).

1 IRAF is distributed by the National Optical Astronomy Observatory, which is operated by the Association of Universities for Research in Astronomy, Inc., under cooperative agreement with the National Science Foundation. 
M. A. Guerrero and L. F. Miranda: NGC 6778: a disrupted planetary nebula around a binary central star
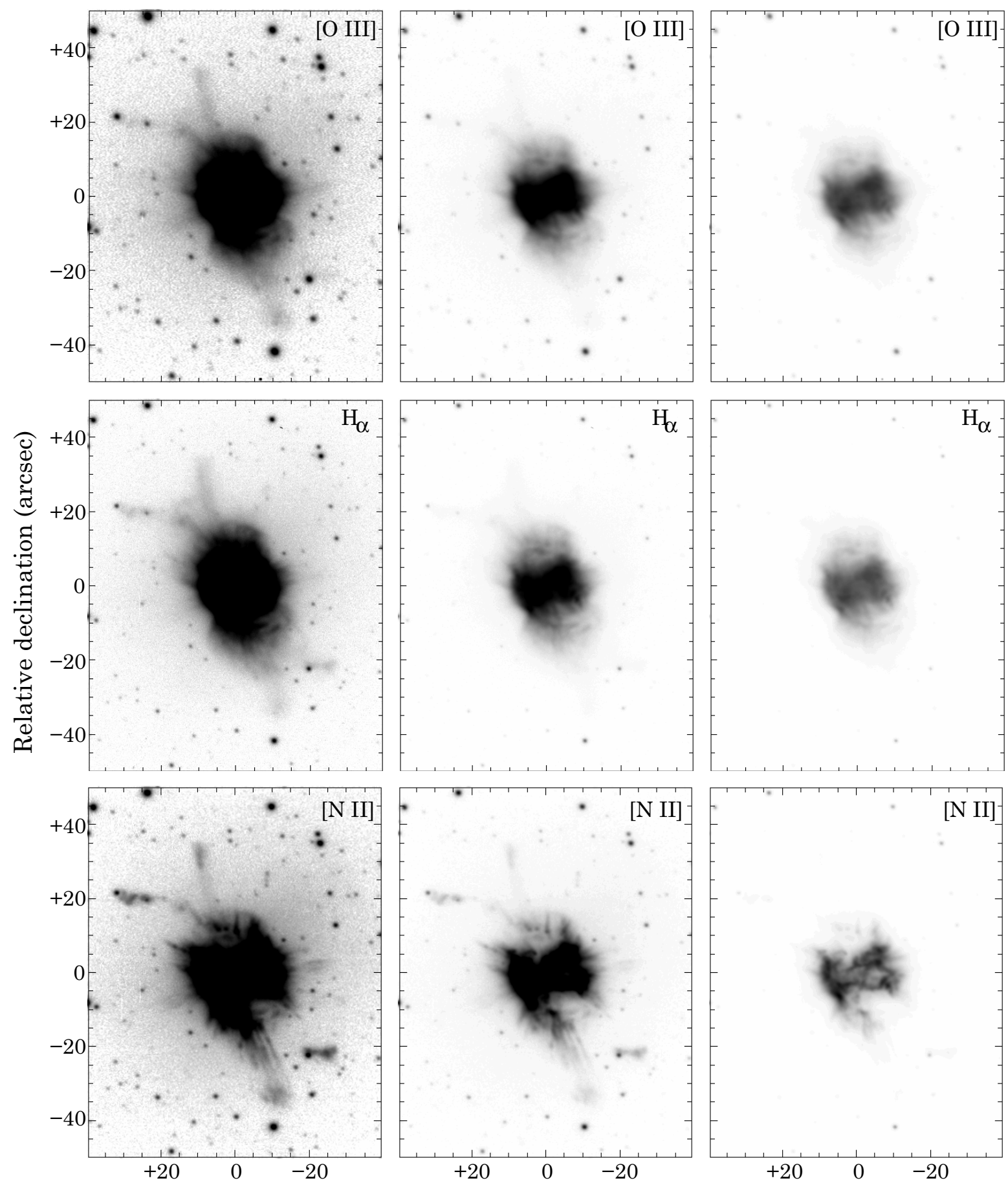

Relative right ascension (arcsec)

Fig. 1. [O III], $\mathrm{H} \alpha$, and [N II] narrow-band images of NGC 6778. The images are shown with three different gray-scales to highlight the faint (left), medium (center), and bright (right) nebular features. The coordinate's origin corresponds to the position of the central star of the nebula, as detected in the near-IR images (see Fig. 2).

\section{Results}

\subsection{The morphology of NGC 6778}

The optical narrow-band images of NGC 6778 (Figs. 1 and 3) provide a sharper view of the morphological components described by Paper I and disclose new interesting features. Additional information on the different morphological components of NGC 6778 and their relative intensities in the $\mathrm{H} \alpha$, [O III], and [N II] emission lines is provided by the composite-color picture shown in Fig. 4 and the $[\mathrm{N} \mathrm{III]/[O} \mathrm{III]} \mathrm{and} \mathrm{H} \alpha /[\mathrm{O}$ III] ratio maps presented in Fig. 5.

\subsubsection{Main nebular shell}

The innermost region of NGC 6778 can be depicted as a highly distorted and fragmented [N II]-bright equatorial ring aligned 

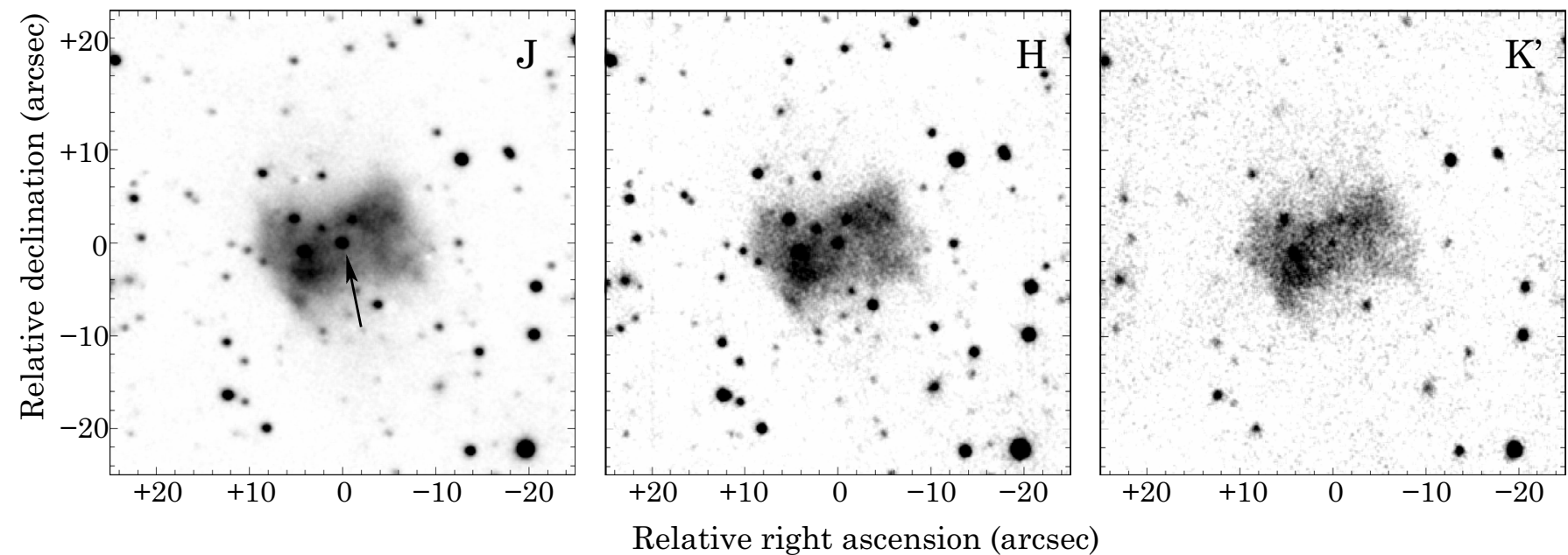

Fig. 2. Gray-scale representation of the near-IR broad-band $J, H$, and $K^{\prime}$ images of NGC 6778. The gray levels are linear. The coordinate's origin corresponds to the position of the central star of the nebula detected in the three filters and marked by an arrow in the $J$ image.

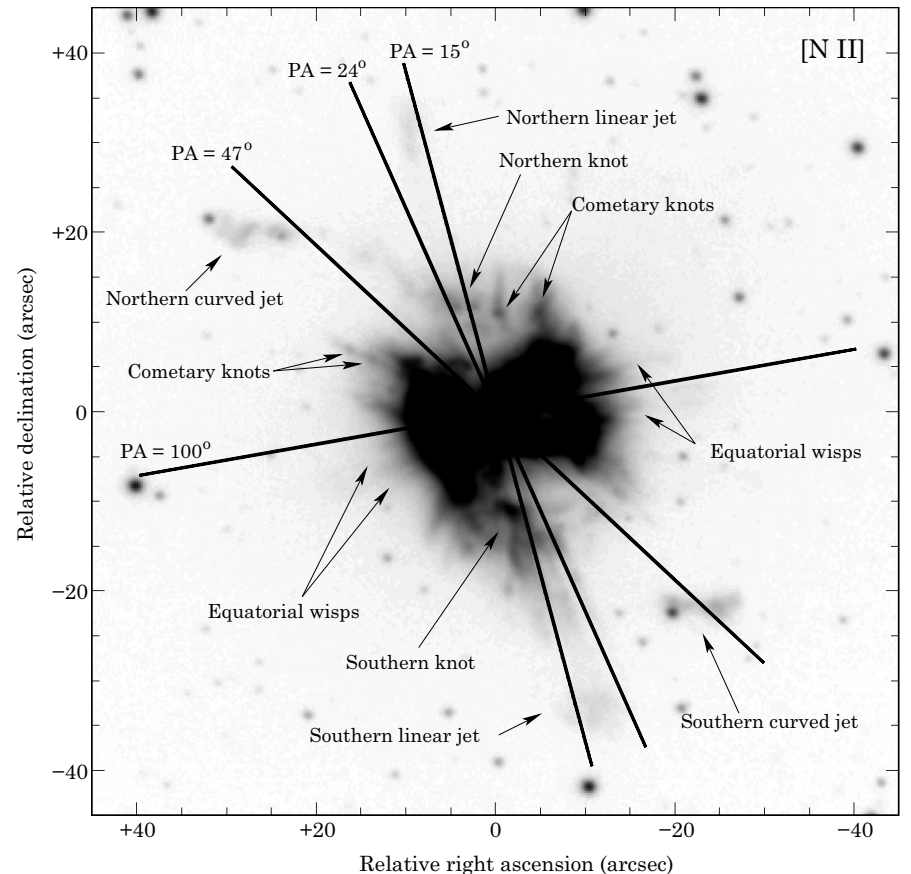

Fig. 3. [N II] image of NGC 6778 overlaid with the positions of the slits used for the acquisition of the high-dispersion echelle spectroscopic observations at CTIO (slit width not to scale). Nebular features are also labeled in the figure.

along a line close to the east-west direction. Although $\mathrm{H} \alpha$ and [O III] emission is also found in these innermost regions, the spatial distribution of the emission from these lines is much smoother than that of $\left[\mathrm{N}_{\mathrm{II}}\right]$. Note also that the values of the $[\mathrm{N}$ II $] /[\mathrm{O}$ III $]$ and $[\mathrm{N}$ II $] / \mathrm{H} \alpha$ ratios peak at the location of this ring. By contrast, the emission in the $\mathrm{H} \alpha$ and $[\mathrm{O} \mathrm{III}]$ lines is brighter along an axis at $\mathrm{PA} \sim 20^{\circ}$, opening in bipolar lobes that span $\sim 17^{\prime \prime}$. As suggested in Paper I, the overall structure of the central region of NGC 6778 can be described as a bipolar PN with a low-ionization ring-like structure embedded within higher excitation bipolar lobes.

The composite-color picture in Fig. 4 and the ratio maps in Fig. 5 reveal clear excitation variations within the inner regions of NGC 6778. At the tips of the equatorial ring, the $\mathrm{H} \alpha$ and

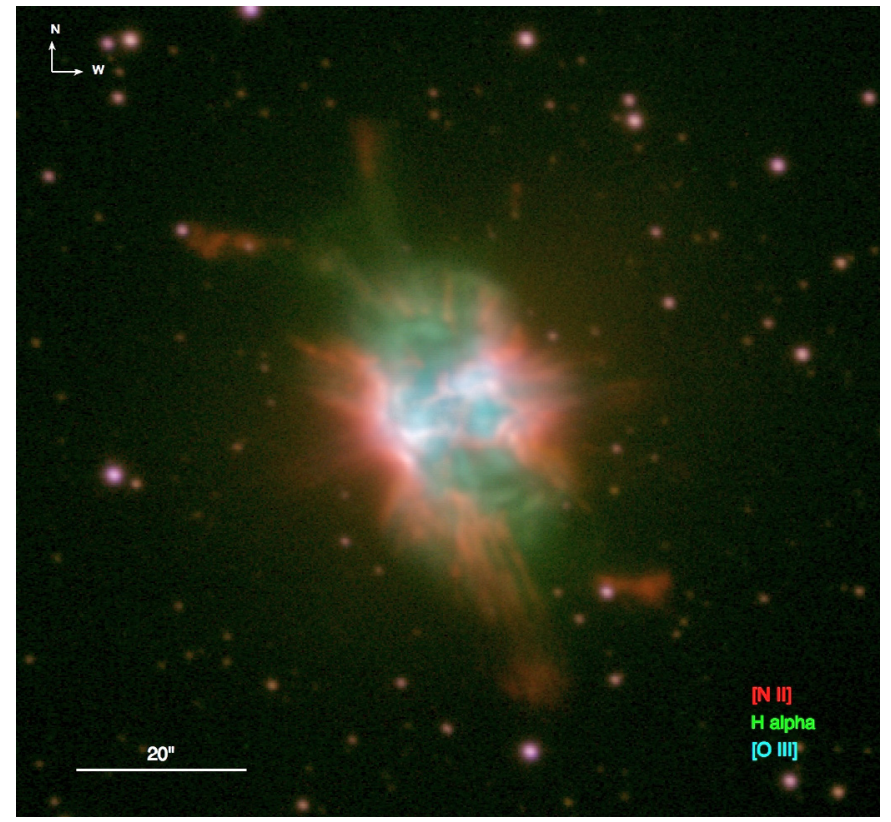

Fig. 4. NOT composite color picture of NGC 6778 in the [O III] (blue), $\mathrm{H} \alpha$ (green), and [N II] (red) emission lines.

[O III] emission is enclosed by bright [N II] emission, resulting in the highest values of the $[\mathrm{N}$ II $] /\left[\mathrm{O}_{\mathrm{III}}\right]$ ratio. This $[\mathrm{N}$ II $]$-bright structure encompasses a spheroidal region in the innermost section of NGC 6778 with the lowest values in the $\mathrm{H} \alpha /[\mathrm{O}$ III] ratio (the bluish central region in the composite-color picture). This [O III]-bright inner region is tipped by two regions of enhanced $\mathrm{H} \alpha /[\mathrm{O} \mathrm{III}]$ ratios along the major axis of the bipolar lobes (seen in greenish colors in the composite-color picture). These changing ratios seem to imply that the nebula is highly ionizationbounded along the equatorial ring, while the bipolar lobes are density-bounded, although some shortage of ionizing photons at their tips is suggested by the enhanced $\mathrm{H} \alpha /[\mathrm{O}$ III $]$ ratio.

The emission in the near-IR images of NGC 6778 (Fig. 2) is mostly limited to the innermost ring-like structure and to the $[\mathrm{N}$ II $]$-bright regions at the tips of this ring. The emission from the bipolar lobes of NGC 6778 is hinted at the $J$ image and, to a lesser extent, at the $H$ image. Given the similarities between the $\mathrm{H} \alpha$ and near-IR images of NGC 6778, we suspect that the 


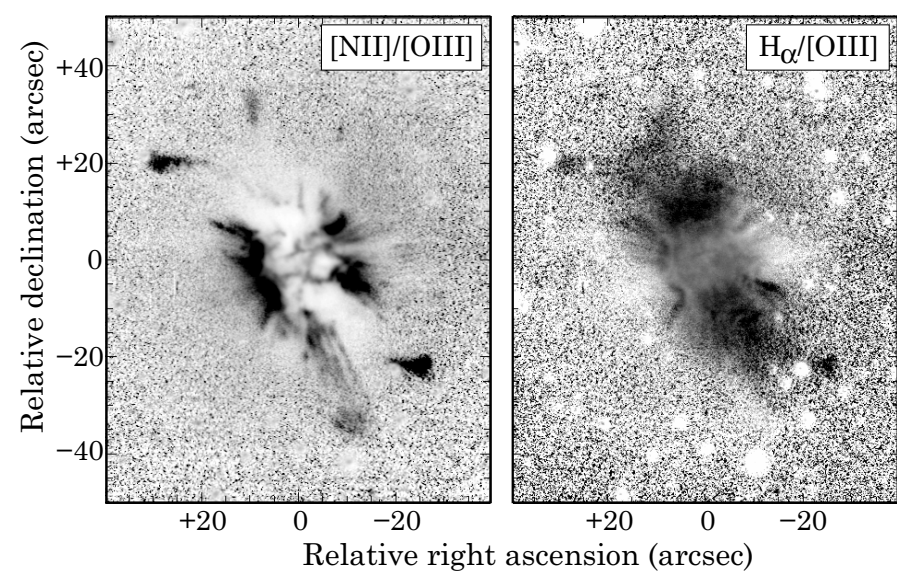

Fig. 5. $[\mathrm{N}$ II $] /\left[\mathrm{O}_{\mathrm{III}}\right]$ and $\mathrm{H} \alpha /\left[\mathrm{O}_{\mathrm{III}}\right]$ ratio maps of NGC 6778. Dark shades correspond to high ratio values.

dominant contribution to the near-IR images are recombination lines of $\mathrm{H}$ I, in particular, the $\mathrm{Pa} \beta \lambda 1.282 \mu \mathrm{m}$ in the $J$ band, the Brackett series in the $H$ band, and the $\operatorname{Br} \gamma 2.166 \mu \mathrm{m}$ in the $K$ band as seen in PNe whose near-IR spectra are dominated by H I lines (Hora et al. 1999).

\subsubsection{Linear and curved jet-like systems}

The NOT optical narrow-band images of NGC 6778 reveal two jet-like systems, as labeled in Fig. 3. These are detected in the three emission lines, but most notably in the [N II] images because they are tipped by bright $[\mathrm{N}$ II] emission caps. We name these two features the linear and curved jet-like systems. The linear jet-like system $(\mathrm{LJ})$ goes along PA $\sim 18^{\circ}$. Its northern component (NLJ) is mostly linear, but it shows a subtle tilt toward lower PA values by the time its [ $\mathrm{N}$ II] emission brightens at radial distances between $\sim 30^{\prime \prime}$ and $36^{\prime \prime}$. 5 . The [N II] image suggests that it originates from a knot marked "Northern knot" in Fig. 3. The southern component of the linear jet (SLJ) consists of a series of filaments that, arising from a bright knot at a radial distance $\sim 10$ " (labeled as "Southern knot" in Fig. 3), run in parallel up to a distance of $\sim 30^{\prime \prime}$, when they brighten into a $3^{\prime \prime} \times 4$ " blob. Given our spatial resolution, it is unclear whether this is a single blob or is composed of individual knots, each of which is associated to one of the linear filaments.

The curved jet-like system has a characteristic S-shape and marked point-symmetric brightness emission. The northern component (NCJ) seems to arise at a radial distance $\sim 17^{\prime \prime}$ and $\mathrm{PA} \sim 35^{\circ}$. Then it steadily increases its PA as it proceeds farther, up to $\mathrm{PA} \sim 55^{\circ}$ at a distance $\sim 39.3$. The emission in the $[\mathrm{N}$ II] line in the linear jet-like system brightens in the region farther away than $\sim 30^{\prime \prime}$ from the nebular center. Indeed, only this bright [ $\left.\mathrm{N}_{\mathrm{II}}\right]$ emission cap is detected for the southern component of the curved jet (SCJ). Both the southern and the northern curved jet-like features are knottier than their corresponding linear counterparts.

\subsubsection{Cometary knots}

A new morphological feature unveiled by the NOT narrow-band images is a group of cometary knots, most prominent in the [N II] image (Fig. 3). These low-excitation knots have bright, compact heads and tails up to $4 "$ long that point outward from the central region of the nebula. Some of them are projected onto the bipolar lobes, while some others are located outside the boundaries of the lobes. It is unclear whether all these knots have a connection with the jet-like systems, but the "Northern knot" and "Southern knot" in Fig. 3 seem to be the origin of the linear jets.

\subsubsection{Equatorial filamentary wisps}

Another interesting feature revealed by the NOT narrow-band images is a system of filamentary wisps protruding mostly from the equatorial regions of the nebula and along radial directions. These wisps are present in all three narrow-band images, but especially in the [N II] and $\mathrm{H} \alpha$ images (Figs. 1 and 3). As the angle between these wisps and the bipolar axis decreases, it becomes unclear whether the radial filamentary structures are wisps or cometary knot tails. Images at a higher angular resolution would be necessary to clearly distinguish wisps from cometary knot tails.

\subsection{Near-IR magnitudes of the central star}

The central star of NGC 6778 has recently been recognized to be in a close binary system (Miszalski et al. 2011). This central star is detected in the broad-band near-IR images of NGC 6778 as the relatively bright star marked by an arrow in the $J$ image in Fig. 2. To derive the near-IR magnitudes of this star, we have compared its instrumental $J H K^{\prime}$ magnitudes with those of seven field stars in our images with $J H K_{\mathrm{s}}$ magnitudes in the 2MASS Point Source Catalogue (PSC, Skrutskie et al. 2006). After the appropriate conversion of $K^{\prime}$ and $K_{\mathrm{s}}$ magnitudes into $K$, this comparison yields $J=16.87 \pm 0.12, H=17.12 \pm 0.09$, and $K=17.38 \pm 0.13$ for the central star, where the $1-\sigma$ errors include the measurement dispersions of the comparison stars and the errors of the measured instrumental magnitudes. These magnitudes imply $J-H=-0.25 \pm 0.15, H-K=-0.26 \pm 0.16$, and $J-K=-0.51 \pm 0.18$ colors. Whereas the value of the $J-H$ color can be expected in hot WDs, we note that the colors derived using the $K$ band are anomalous. In particular, the value of the $J-K$ color is only marginally consistent with the values in the range from -0.20 to -0.30 mag expected for a WD (Zuckerman et al. 1991; Green et al. 2000), and it seems to imply a lack of emission in the $K$ band (or an excess of emission in the $J$ band). We attribute this odd value to the difficulties in the measurement of the $K$ magnitude posed by the faintness of the star in this band and by the subtraction of the contribution of adjacent nebular emission.

The lack of near-IR excess and the observed JHK magnitudes can be used to provide a crude constraint to the spectral type and luminosity class of the companion to the CSPN of NGC 6778. A giant or sub-giant companion can be definitely discarded, but a dwarf companion is possible. The latest spectral type of this putative dwarf companion depends on the distance to the nebula: $\mathrm{K} 0 \mathrm{~V}$ for a distance to the nebula $\leq 4 \mathrm{kpc}, \mathrm{M} 0 \mathrm{~V}$ for a distance $\leq 2 \mathrm{kpc}$, and M5 V for a distance $\leq 1 \mathrm{kpc}$.

\subsection{Spatio-kinematical properties of NGC 6778}

The preliminary description of the overall kinematics of NGC 6778 reported in Paper I revealed a bipolar structure for the bright nebular shell, whereas the two pairs of jet-like features have high expansion velocities that increase outward, i.e., they are real fast collimated outflows. Here we revisit these $\mathrm{H} \alpha$ and [N II] $\lambda 6583$ echellograms (Fig. 6) in the light of the improved spatial resolution of the NOT narrow-band images, 

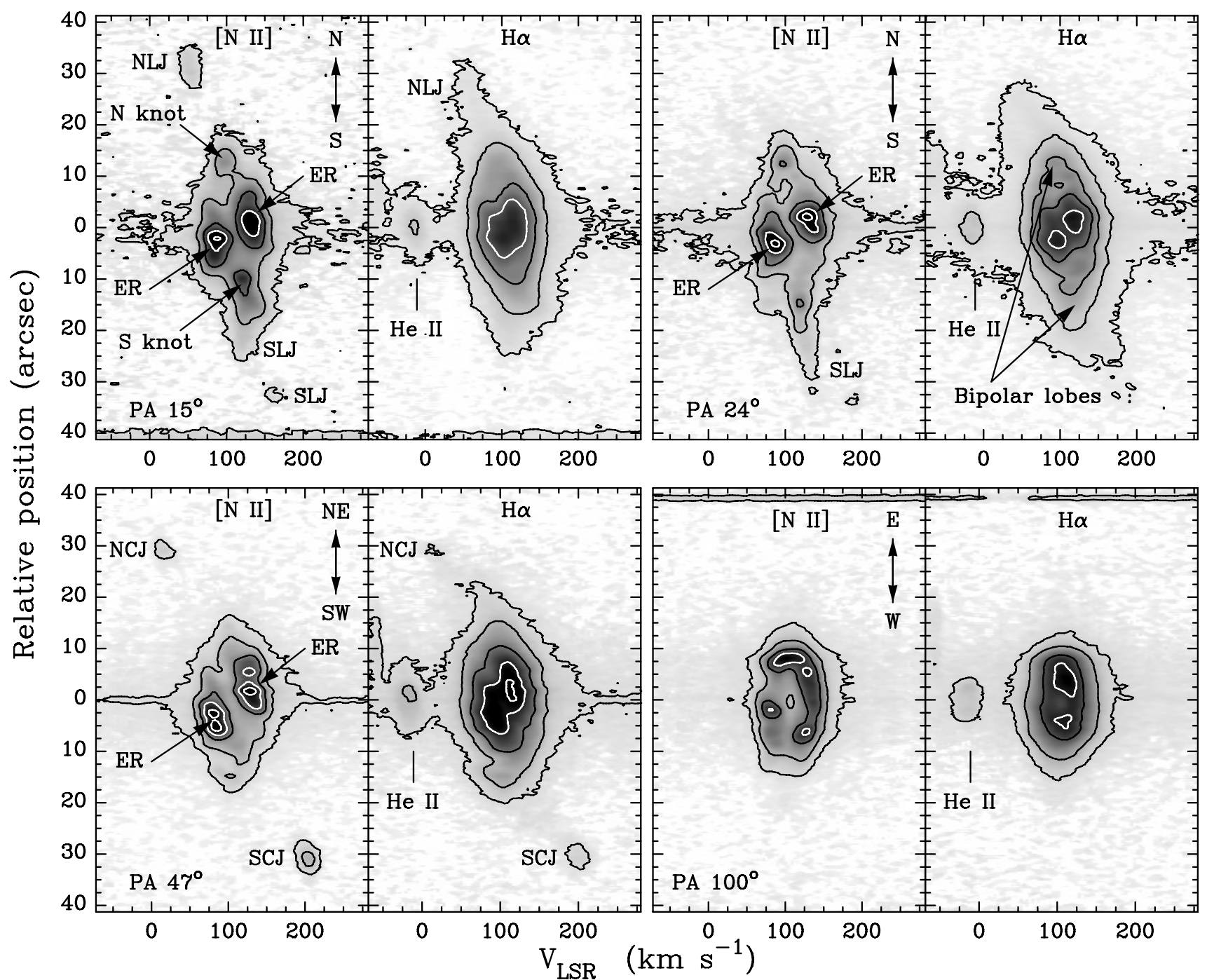

Fig. 6. Gray-scale position-velocity (PV) echellograms of the $\mathrm{H} \alpha$ and [N II] emission lines of NGC 6778 observed at four slits along the PA's $15^{\circ}$, $24^{\circ}, 47^{\circ}$, and $100^{\circ}$. Note the presence of emission from the He II $\lambda 6560$ line redward of $\mathrm{H} \alpha$. The morphological and kinematical features of interest are labeled on different echellograms: the northern linear jet (NLJ), the southern linear jet (SLJ), the northern curved jet (NCJ), the southern curved jet (SCJ), the equatorial ring (ER), the bipolar lobes, and the north knot (N knot) and south knot (S knot). The contour levels have been selected to emphasize the different features.

placing special emphasis on the kinematics of individual spatiokinematical features.

\subsubsection{Equatorial ring}

First, we note that the echellograms of the $[\mathrm{N}$ II $]$ line from slits close to the symmetry axis (PA's $15^{\circ}, 24^{\circ}$, and $47^{\circ}$ ) show bright emission peaks detected at short radial distances. These emission peaks trace the emission from the distorted equatorial ring described in the images and were consequently labeled "ER" in Fig. 6. According to these echellograms, the northern regions of the equatorial ring recede from us, while the southern regions move toward us. The echellograms at PAs $15^{\circ}$ and $24^{\circ}$ clearly reveal arc-like features departing from these peaks with increasing radial velocity. This can be interpreted as the opening of the ring or toroidal structure into the wider, higher velocity structure of the bipolar lobes, although it may alternatively be associated to the radial filaments of cometary knots and/or equatorial wisps.

The $\left[\mathrm{N}_{\text {III }}\right.$ echellogram of the slit at PA $100^{\circ}$ reveals a donutshaped line that is indicative of the expansion of the equatorial ring $^{2}$, while the $\mathrm{H} \alpha$ echellogram shows a rather filled line with two peaks closer than the edges of the $[\mathrm{N} \mathrm{II}]$ line. The velocity split derived from the $[\mathrm{N} \mathrm{II}]$ position-velocity map $\left(52.2 \mathrm{~km} \mathrm{~s}^{-1}\right)$ would imply an expansion velocity $\sim 26 \mathrm{~km} \mathrm{~s}^{-1}$, although it must be noted that the line-shape is not exactly an ellipse. These distortions of the velocity field are confirmed by the velocity split derived for the ring from the echellograms at the other PAs: $43.5 \mathrm{~km} \mathrm{~s}^{-1}$ at PA $15^{\circ}, 40.6 \mathrm{~km} \mathrm{~s}^{-1}$ at PA $24^{\circ}$, and $49.3 \mathrm{~km} \mathrm{~s}^{-1}$ at PA $47^{\circ}$. In view of the distorted morphology and kinematics of the ring, we must admit that the inclination angle of $15^{\circ}$ derived in Paper I under the assumption of a circular cross-section is questionable.

\subsubsection{Bipolar lobes}

Next we note the bipolar lobes, best seen in the $\mathrm{H} \alpha$ echellograms along PAs $15^{\circ}$ and $24^{\circ}$ because they are fainter in the

2 The higher surface brightness of the blue-shifted (northern) section of this line is most likely caused by the slit position passing closer to the northern section of the ring. 
M. A. Guerrero and L. F. Miranda: NGC 6778: a disrupted planetary nebula around a binary central star

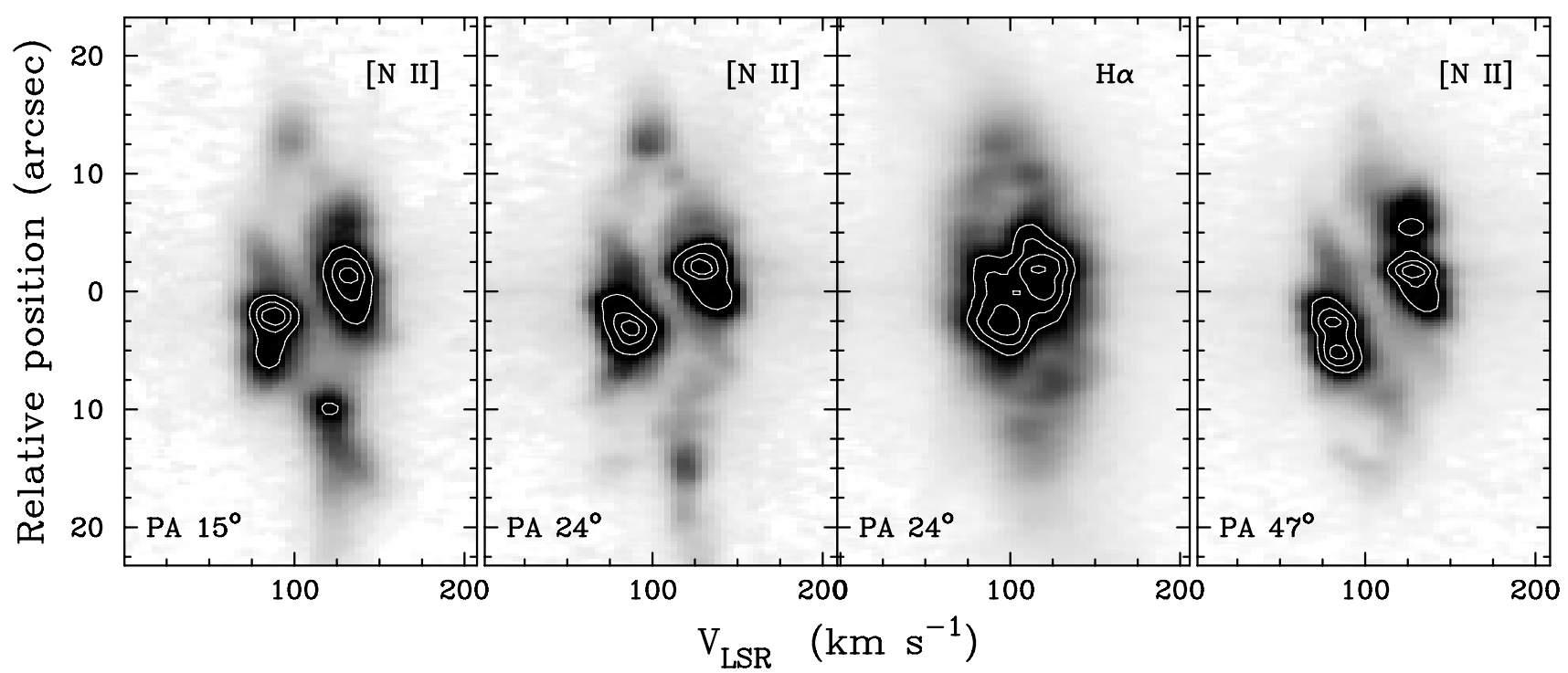

Fig. 7. Gray-scale PV maps of $\mathrm{H} \alpha$ at $\mathrm{PA} 24^{\circ}$, and $[\mathrm{N} \mathrm{II}]$ at PA $15^{\circ}, 24^{\circ}$, and $47^{\circ}$, where the gray levels have been chosen to highlight the kinematics of the bipolar lobes.

low-ionization [N $\mathrm{NI}]$ line. The bipolar axis is tilted such that the north lobe is blue-shifted, conforming to the inclination of the equatorial ring. The difference in velocity between the tips of the north and south lobes is $\sim 20 \mathrm{~km} \mathrm{~s}^{-1}$. Since the velocity split of the equatorial ring is larger than the velocity difference between the bipolar tips, it indicates that the inclination angle of the bipolar axis with respect to the plane of the sky is small. If we assume a similar expansion, i.e., the expansion velocity increases with radial distance, we can derive an inclination angle $\sim 12^{\circ}$ and a deprojected expansion velocity at the tip of the bipolar lobes of $50 \mathrm{~km} \mathrm{~s}^{-1}$.

A close inspection of the internal kinematics of the bipolar lobes (Fig. 7) reveals that it differs from the typical hourglass-like expansion observed in other bipolar PNe (e.g., K 4-55; Guerrero et al. 1996). Most notably, the [N II] PV maps at PA $15^{\circ}$ and $24^{\circ}$ show a steady increase of the velocity split between the front and rear walls of the bipolar lobes up to a distance $\sim 7^{\prime \prime}$, but, at greater angular distances, the velocity split abruptly decreases to rapidly converge into the $\mathrm{N}$ and $\mathrm{S}$ knots. Moreover, both the [N II] and $\mathrm{H} \alpha$ PV maps at these PAs show the emission from the bipolar lobes delineating a twisted cavity (Fig. 7).

There is also evidence that implies that the bipolar lobes have been bored along selected directions. At PA $24^{\circ}$, the front side of the southwestern lobe shows a breaking in the emission line at the position of a knot located at $15^{\prime \prime}$ and $+28 \mathrm{~km} \mathrm{~s}^{-1}$, i.e., the same projected distance as the $\mathrm{S}$ knot but at a different radial velocity. The PV map of the [N II] line at PA $47^{\circ}$ also shows openings at its tips, as well as a hook-shaped structure southward between $9^{\prime \prime}$ and $18^{\prime \prime}$ and radial velocities between $+17 \mathrm{~km} \mathrm{~s}^{-1}$ and $-20 \mathrm{~km} \mathrm{~s}^{-1}$ that is hard to reconcile with an hour-glass-like shell. These results suggest that, similarly to the equatorial region, the bipolar lobes are disrupted and their kinematics distorted. That the images show morphologically well defined lobes while their kinematics is highly complex may suggest that the disruption of the lobes is a relatively recent phenomenon that occurred on a time-scale shorter than the process that formed the bipolar shell.

\subsubsection{Linear and curved jets}

The echellograms displayed in Fig. 6 clearly confirm that the two jet-like systems described in Sect. 3.1 are associated with high-velocity features. The radial velocity of the linear jets increases steadily with the distance to the central star of NGC 6778 up to a maximum velocity split $\Delta v=113 \mathrm{~km} \mathrm{~s}^{-1}$. Similarly, the curved jets show a velocity split $\Delta v=192 \mathrm{~km} \mathrm{~s}^{-1}$. In both cases, the FWHM of the lines are very narrow, $\sim 23 \mathrm{~km} \mathrm{~s}^{-1}$ at the tip of NLJ, and $\sim 16 \mathrm{~km} \mathrm{~s}^{-1}$ for the tips of SCJ and NCJ. If we assume an inclination angle of $12^{\circ}$ with respect to the plane of the sky, as for the bipolar lobes, then the deprojected velocities are $270 \mathrm{~km} \mathrm{~s}^{-1}$ for the linear jet, and $460 \mathrm{~km} \mathrm{~s}^{-1}$ for the curved jet. We note that the assumption that these collimated outflows share the same inclination as the bipolar shell is questionable, most especially for the curved jet. At any rate, the jet-like morphology of these features, their high systemic velocities and their low dispersion in velocity lead us to conclude that they are true fast collimated outflows that deserve to be called jets.

We note that no expansion was registered between the 2005 August and 2008 August images. Assuming conservatively that it would have been possible to measure a position shift $\sim 20 \%$ the spatial resolution, i.e., $\sim 0$ ' 16 , the expansion velocity of the linear jet along the plane of the sky sets a lower limit for the distance to NGC 6778 of $\geq 1 \mathrm{kpc}$.

The kinematics of the faintest features associated to these jets is best illustrated in Fig. 8. The PV map of the [N II] line at PA $24^{\circ}$ shows a faint knot, coincident with the blob at the head of the SLJ, $\simeq 35^{\prime \prime}$ apart from the central star, which exhibits a radial velocity with respect to the system of $+83 \mathrm{~km} \mathrm{~s}^{-1}$, much higher than the radial velocity at the tip of the jet, $\simeq 56 \mathrm{~km} \mathrm{~s}^{-1}$. If this knot moved at an angle of $12^{\circ}$ with respect to the plane of the sky, its velocity would be $400 \mathrm{~km} \mathrm{~s}^{-1}$, i.e., higher than the velocity derived for LJ. This behavior may be indicative of an acceleration of SLJ at long distances, although the abrupt velocity increase results more likely from a change of the direction of the outflow that would be pointing more closely to the line of sight at this location. In the latter case, the abrupt change in direction could be tracing the interaction of SLJ with an ambient clump. We note that this phenomenon is not observed in the NLJ and CJ.

The complex kinematics in the region of $\mathrm{LJ}$ is best seen in the PV map of the $\mathrm{H} \alpha$ line at PA $24^{\circ}$ (Fig. 8). The $\mathrm{H} \alpha$ emission shows a bubble-like structure surrounding the emission from the NLJ. This bubble extends from $\simeq 12^{\prime \prime}$ up to $\simeq 30^{\prime \prime}$, 


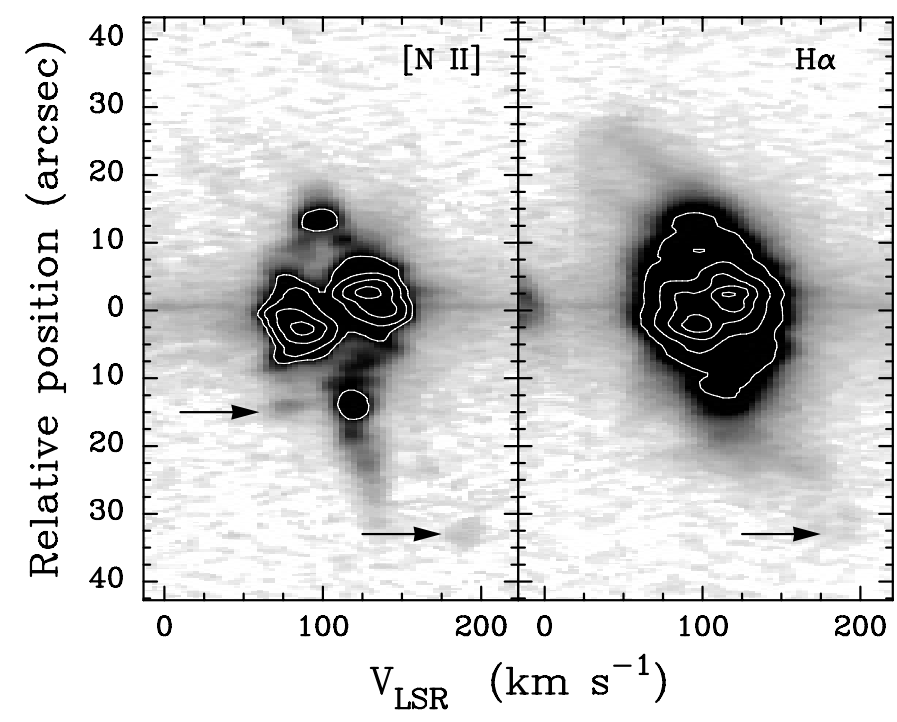

Fig. 8. Gray-scale PV maps of the $\mathrm{H} \alpha$ and $\left[\mathrm{N}\right.$ II] emissions at PA $24^{\circ}$. The arrows mark the location of knots described in Sect. 3.3.3.

is mainly shifted toward the blue (as it is NLJ), and presents a maximum velocity width $\simeq 100 \mathrm{~km} \mathrm{~s}^{-1}$. A similar structure might be associated to SLJ, as suggested by the high velocity width $\left(\simeq 110 \mathrm{~km} \mathrm{~s}^{-1}\right)$ of the preferentially red-shifted, faint emission found at $\simeq 24^{\prime \prime}$ from the center. Similar structures are not associated to the CJ. However, while the $\left[\mathrm{N}_{\mathrm{II}}\right]$ emission is found mostly at the northern and southern heads of CJ, faint $\mathrm{H} \alpha$ emission connects the heads of $\mathrm{CJ}$ with the outer regions of the bipolar lobes (see PA $47^{\circ}$ in Fig. 6). This faint $\mathrm{H} \alpha$ emission reveals an outward increase of the radial velocity along the CJ.

\section{Discussion}

The observational results presented in the previous section provide compelling evidence of strong dynamic interactions affecting the different nebular components of NGC 6778. Like in NGC 6326, which is described by Miszalski et al. (2011), the equatorial ring of NGC 6778 exhibits a highly distorted shape and morphological and kinematical signs of disruption. The equatorial wisps and cometary knots are also indicative of material being eroded from these knots and blown away. A fast stellar wind, a sudden mass ejection event, or the ablation by UV photons of dense knots may be responsible for the observed morphological features.

Similarly, the complex kinematics of the bipolar lobes suggests a dynamical interaction that has distorted its kinematics. There are interesting similarities with NGC 6572 (Miranda et al. 1999b), whose bipolar lobes also present a complex kinematics that cannot be interpreted with a simple (ellipsoidal o bipolar) geometry. There is also a connection between the collimated outflows of NGC 6778 and some bright knots at the tips of the bipolar shell, as seen in other PNe (e.g., NGC 6891; Guerrero et al. 2000). Very notably, the SLJ component seems to originate from a bright knot that is located in the PV plots at the point where the blue and red components of the bipolar lobes merge. The connection between the jets and knots at the tips of the bipolar lobes suggests either that the collimation of the outflows occurred at the tip of the bipolar lobes (as in the converging flows scenario envisaged by Frank et al. 1996) or that the interaction of the fast collimated outflows have shaped the bipolar shell (as suggested by Sahai \& Trauger 1998). The relatively undisturbed morphology of the bipolar lobes, in contrast with its kinematical properties, suggests that the bipolar lobes have only recently been shocked by the collimated outflows, i.e., that the bipolar lobes are older than the collimated outflows, formed in a late ejection event. The hole-like features of the bipolar lobes on the PV map at PA $47^{\circ}$ (Fig. 7), the direction along which the curved jets are detected, provides strong support for a lobe-jet interaction.

In this context, a comparison between the kinematical ages of shells and collimated outflows may be of interest to determine the time line of the formation of NGC 6778, although the many dynamical effects involved and the uncertainty in their inclination can affect noticeably their absolute values (Corradi et al. 1999). We derive a kinematical age $\sim 1700 \times d$ yr for the equatorial ring, $\sim 1600 \times d \mathrm{yr}$ for the bipolar lobes, and $\sim 650 \times d$ yr for the linear jet, where $d$ is the distance in kpc. The similar kinematical ages of the equatorial ring and bipolar lobes can be expected if they formed close in time. If we assume that the equatorial ring and nebular shell are indeed coeval and formed some $1700 \times d \mathrm{yr}$ ago, then it can be envisaged that a more recent, $\sim 650 \times d$ yr old, series of collimated outflows interacted with the nebular shell.

The increasing expansion velocity of the collimated outflows with radius also suggests a free expansion that can be associated with a unique and sudden ejection event. There are several cases of this type of explosive-like, late events in PNe, especially associated to the late helium-shell flash associated to the bornagain class of PNe (Guerrero \& Manchado 1996; Wesson et al. 2008) or to oxygen-neon-magnesium nova-like events suggested for the born-again PN A 58 (Lau et al. 2011) or for BD+30³639 (Maness et al. 2003). As was to be expected after a CE evolution (Iben \& Tutukov 1993), the chemical composition of NGC 6778 is typical of type I bipolar PNe (Milingo et al. 2010), with no indications of neon overabundances or hydrogen-poor inclusions, although we note that these chemical abundances most likely correspond to the brightest regions of the main nebula.

Finally, let us assess whether the nebular inclination is consistent with the inclination of the orbital plane of the binary system at the central star of NGC 6778 required to eclipse the primary star. If we adopt a $0.6 M_{\odot}$ central star and a $0.3 M_{\odot}$ dwarf companion of spectral type M4, which is not discarded by the near-IR data presented in Sect. 3.2, the short orbital period of $\sim 0.15$ days would imply a semimajor axis of $\sim 0.005$ AU. For such a small orbital separation, it can be shown that the inclination angle of $12^{\circ}$ of the bipolar lobes ${ }^{3}$ would still produce the eclipses of the primary star observed in the lightcurve of the central star.

\section{Summary}

We have analyzed the detailed morphology and kinematics of NGC 6778, a PN whose central star is in a binary system with a short orbital period, $\sim 0.15$ days. The main nebular shell consists of a disrupted equatorial ring and kinematically disturbed bipolar lobes. The idea that the chaotic morphology and kinematics of NGC 6778 stems from a violent event of sudden mass ejection is reinforced by the presence of equatorial wisps and cometary knots. There is an intimate connection between the fast collimated outflows and several kinematical and morphological features of the bipolar lobes. All these facts suggest that NGC 6778 may have experienced a late explosive event that produced fast collimated outflows whose interaction with the nebular envelope

\footnotetext{
Miszalski et al. (2011) quoted Paper I for an inclination of $5^{\circ}$ that should have read $15^{\circ}$.
} 
has had dramatic effects in the nebular shaping. There is, however, no additional evidence for a late helium shell flash or a nova-like explosion.

Acknowledgements. Part of this work was supported by projects AYA2008-01934 and AYA2011-29754-C03-02 of the Spanish MICINN (co-funded by FEDER funds). L.F.M. acknowledges partial support from INCITE09 E1R312096, INCITE09312191PR, and IN8458-2010/061 of Xunta de Galicia (co-funded by FEDER funds). We thank the group of support astronomers of the Instituto de Astrofísica de Canarias (IAC) for making available the narrow-band filters used at the NOT observations. We also thank G. Ramos-Larios for his assistance during the 2008 August NOT run and for the preparation of the color picture of NGC 6778.

\section{References}

Balick, B. 1987, AJ, 94, 671

Balick, B., \& Frank, A. 2002, ARA\&A, 40, 439

Blackman, E. G. 2009, in Cosmic Magnetic Fields: From Planets, to Stars and Galaxies, ed. K. G. Strassmeier, A. G. Kosovichev, \& J. E. Beckman (Dordrecht: Kluwer), IAU Symp., 259, 35

Bond, H. E., \& Livio, M. 1990, ApJ, 355, 568

Borkowski, K. J., Blondin, J. M., \& Harrington, J. P. 1997, ApJ, 482, L97

Bujarrabal, V., Alcolea, J., Sánchez Contreras, C., \& Sahai, R. 2002, A\&A, 389, 271

Cantó, J., Tenorio-Tagle, G., \& Rozýczka, M. 1988, A\&A, 192, 287

Corradi, R. L. M., Perinotto, M., Villaver, E., Mampaso, A., \& Gonçalves, D. R. 1999, ApJ, 523, 721

Durand, S., Acker, A., \& Zijlstra, A. 1998, A\&AS, 132, 13

Frank, A., Balick, B., \& Livio, M. 1996, ApJ, 471, L53

García-Segura, G., López, J. A., \& Franco, J. 2005, ApJ, 618, 919

Green, P. J., Ali, B., \& Napiwotzki, R. 2000, ApJ, 540, 992

Guerrero, M. A., \& Manchado, A. 1996, ApJ, 472, 711

Guerrero, M. A., \& Manchado, A. 1998, ApJ, 508, 262

Guerrero, M. A., Manchado, A., \& Serra-Ricart, M. 1996, ApJ, 456, 651

Guerrero, M. A., Miranda, L. F., Manchado, A., \& Vázquez, R. 2000, MNRAS, 313,1

Guerrero, M. A., Miranda, L. F., Chu, Y.-H., Rodríguez, M., \& Williams, R. M. 2001, ApJ, 563, 883

Hora, J. L., Latter, W. B., \& Deutsch, L. K. 1999, ApJS, 124, 195
Iben, I., Jr., \& Tutukov, A. V. 1993, ApJ, 418, 343

Kwok, S. 1983, in Planetary Nebulae, ed. D. R. Flower (Cambridge: Cambridge Univ. Press), IAU Symp. 103, 293

Kwok, S., \& Su, K. Y. L. 2005, ApJ, 635, L49

Lau, H. H. B., De Marco, O., \& Liu, X.-W. 2011, MNRAS, 410, 1870

Livio, M. 2000, in Asymmetrical Planetary Nebulae II: From Origins to Microstructures, ed. J. H. Kastner, N. Soker, \& S. Rappaport (San Francisco: ASP), ASP Conf. Ser., 199, 243

Maestro, V., Guerrero, M.A., \& Miranda, L. F. 2004, in Asymmetrical Planetary Nebulae III: Winds, Structure and the Thunderbird, ed. M. Meixner, J. H. Kastner, B. Balick, \& N. Soker (San Francisco: ASP), ASP Conf. Ser., 313, 127 (Paper I)

Maness, H. L., Vrtilek, S. D., Kastner, J. H., \& Soker, N. 2003, ApJ, 589, 439

Milingo, J. B., Kwitter, K. B., Henry, R. B. C., \& Souza, S. P. 2010, ApJ, 711, 619

Miranda, L. F., Guerrero, M. A., \& Torrelles, J. M. 1999a, AJ, 117, 1421

Miranda, L. F., Vázquez, R., Corradi, R. L. M., et al. 1999b, ApJ, 520, 714

Miranda, L. F., Ramos-Larios, G., \& Guerrero, M. A. 2010, PASA, 27, 180 (Paper II)

Miszalski, B. 2012, in Planetary Nebulae, an Eye to the Future, ed. A. Manchado, D. Schönberner, \& L. Stanghellini (Cambridge: Cambridge Univ. Press), IAU Symp. 283, in press

Miszalski, B., Jones, D., Rodríguez-Gil, P., et al. 2011, A\&A, 531, A158

Morris, M. 1987, PASP, 99, 1115

Nordhaus, J., \& Blackman, E. G. 2006, MNRAS, 370, 2004

Nordhaus, J., Blackman, E. G., \& Frank, A. 2007, MNRAS, 376, 599

Patriarchi, P., \& Perinotto, M. 1991, A\&AS, 91, 325

Perinotto, M., Patriarchi, P., Balick, B., \& Corradi, R. L. M. 2004, A\&A, 422, 963

Riera, A., García-Lario, P., Manchado, A., Bobrowsky, M., \& Estalella, R. 2003, A\&A, 401, 1039

Sahai, R. 2000, ApJ, 537, L43

Sahai, R., \& Nyman, L.-A. 2000, ApJ, 538, L145

Sahai, R., \& Trauger, J. T. 1998, AJ, 116, 1357

Schwarz, H. E., Corradi, R. L. M., \& Melnick, J. 1992, A\&AS, 96, 23

Skrutskie, M. F., Cutri, R. M., Stiening, R., et al. 2006, AJ, 131, 1163

Soker, N. 2004, in Asymmetrical Planetary Nebulae III: Winds, Structure and the Thunderbird, ed. M. Meixner, J. H. Kastner, B. Balick, \& N. Soker (San Francisco: ASP), ASP Conf. Ser., 313, 562

Wesson, R., Barlow, M. J., Liu, X.-W., et al. 2008, MNRAS, 383, 1639

Zuckerman, B., Becklin, E. E., \& McLean, I. S. 1991, in Astrophysics with Infrared Arrays, ed. R. Elston (San Francisco: ASP), ASP Conf. Ser., 14, 161 